\title{
Impacto de la COVID-19 en la consecución del desarrollo sostenible: Una Revisión
}

\section{Impact of COVID-19 on achieving sustainable development: A Review}

\author{
Víctor Gómez ${ }^{1, a, *}$, Hurley Quispe-Ccasa ${ }^{1, b}$ \\ ${ }^{1}$ Programa de Doctorado en Ciencias para el Desarrollo Sustentable, Escuela de Posgrado, Universidad Nacional Toribio \\ Rodríguez de Mendoza, Chachapoyas, Amazonas, Perú.

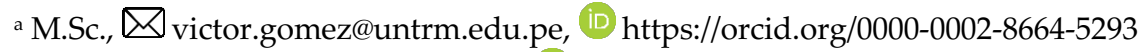

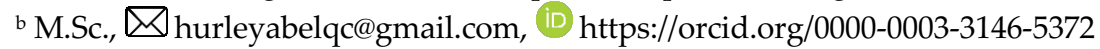 \\ * Autor de Correspondencia: Tel. +51 954811318 \\ http://dx.doi.org/10.25127/riagrop.20213.699
}

http://revistas.untrm.edu.pe/index.php/RIAGROP revista.riagrop@untrm.edu.pe

Recepción: 25 de marzo 2021

Aprobación: 26 de mayo 2021

Este trabajo tiene licencia de Creative Commons. Attribution-NonCommercial-ShareAlike $\quad 4.0$ International Public License - CC-BY-NC-SA 4.0

\section{Resumen}

La aparición de la COVID-19 ha generado una crisis en el planeta. Muchos países han adoptado medidas para evitar el contagio y disminuir su impacto en la población, como el aislamiento social obligatorio. Casi inmediatamente, esta situación se ha volcado en una crisis económica y social por la interrupción de las actividades productivas y el confinamiento de la población. En este estudio de revisión, se analizan los impactos generados directa e indirectamente por la pandemia, desde la perspectiva económica, ambiental y social; de acuerdo a los objetivos propuestos para el logro del desarrollo sostenible. Aunque muchas entidades se propusieron acciones para atenuar los impactos económicos y sociales, y sostener el actual sistema de desarrollo; es importante reconocer las lecciones que nos deja la crisis mundial para encaminar un modelo de desarrollo sostenible, que incluya el abordaje de las desigualdades entre países y grupos sociales, así como el deterioro medioambiental.

Palabras clave: COVID-19, pandemia, aislamiento social obligatorio, recesión mundial, desarrollo sostenible. 


\begin{abstract}
The appearance of COVID-19 has generated a crisis on the planet and many countries have adopted measures to avoid contagion and lessen its impact on the population, such as forced social isolation. Almost immediately, it has turned into an economic and social crisis due to the interruption of productive activities and the confinement of the population. This review study analyzes the impacts generated directly and indirectly by the pandemic, viewed from the economic, environmental and social dimensions; according to the objectives proposed for the achievement of sustainable development. Although many entities propose actions to alleviate the economic and social impacts, and sustain the current development system; It is important to recognize the lessons that the global crisis leaves us to guide a sustainable development model that includes addressing inequalities between countries and social groups, as well as environmental deterioration.
\end{abstract}

Keywords: COVID-19, pandemic, forced social isolation, global recession, sustainable development.

\section{INTRODUCCIÓN}

A nivel mundial, la crisis sanitaria de la pandemia de la COVID-19, ocasionada por el virus SARS-CoV-2 (Severe Acute Respiratory Syndrome Coronavirus 2), se ha tornado en crisis humana y económica sin precedentes. Por ello, las medidas para combatirla se modifican constantemente. Esta enfermedad tuvo su primer registro en la ciudad de Wuhan (China) el 31 de diciembre de 2019 (OMS, 2020) y, en pocos días, se tornó en una amenaza para la mayoría de países. En Perú, con fecha 6 de marzo de 2020, se reportó el primer caso de COVID-19 y el 15 de marzo se decretó el estado de Emergencia Nacional y el aislamiento social obligatorio para detener el avance de contagios.

A la fecha, se desconoce la duración de la emergencia sanitaria, así como la manera en la que se desenvolverá la población en el escenario pospandemia. Lo que es evidente son los impactos que han generado, en el corto plazo, la enfermedad emergente y sus medidas de contención, similares a contextos de guerra, como la interrupción de las actividades económicas y el confinamiento de las poblaciones, que entraron en periodos de cuarentena más o menos severas en la mayoría de países (CEPAL, 2020). Se espera que los países con mejor estructura sanitaria $y$ financiera estén en mejor posición defensiva que los países más débiles y menos desarrollados, para enfrentar la casi inexorable crisis económica y social (Valle et al., 2020). Sin embargo, esta pandemia ha sido catalizada por los efectos de la globalización de las actividades humanas como la depredación de la biodiversidad, el transporte aéreo, el comercio transcontinental, el turismo en masa, entre otros. Según Valle et al. (2020), el actual sistema de globalización se ha centrado, estructuralmente, en factores económicos que relegan los elementos socioculturales $y$ ambientales, igual de relevantes. Por el contrario, este sistema promueve la depredación ambiental y segregación social, en un curso de aceleración entrópica, probablemente inevitable. Por ello, muchos autores coinciden que este escenario de crisis constituye una lección y, a la vez, una valiosa oportunidad para replantear el modelo de desarrollo económico actual, hacia la integración entre los sectores económico, social, cultural, ambiental, tecnológico y político, en 
virtud de la consecución del desarrollo sostenible.

El desarrollo sostenible, en esencia, es aquel modelo de desarrollo en el que se satisfacen las necesidades de la sociedad actual, sin comprometer las necesidades de las futuras generaciones. Por ello, este paradigma de desarrollo solo podrá ser sostenible si las decisiones económicas están vinculadas, básicamente, con el bienestar social y ecológico (Sánchez, 2010). En este estudio de revisión, se abordan los impactos negativos y positivos de la crisis instaurada por el contexto de pandemia de la COVID-19 y cómo influye en una visión para el logro de un modelo de desarrollo sostenible.

\section{IMPACTOS ECONÓMICOS}

En nuestro actual sistema de globalización, muchas consecuencias económicas, ambientales, socioculturales y políticas están relacionadas con la posibilidad de surgimiento y expansión acelerada de nuevas enfermedades. Los factores que generan las condiciones adecuadas para potenciar esta posibilidad son las elevadas tasas de crecimiento poblacional, gran desplazamiento humano, incontrolable explotación de los recursos naturales, mala educación en temas sanitarios, inestabilidad social e incremento de la contaminación (Arias et al., 2020). Por ello, en el escenario actual de expansión global o pandemia por la COVID-19, la mayoría de gobiernos han adoptado el autoaislamiento, la cuarentena y el distanciamiento social obligatorio que trae, como consecuencia inmediata, la desaceleración de la producción y en algunos casos hasta su interrupción total (CEPAL, 2020).
Sin embargo, es necesario saber que el desempeño de la economía y comercio mundial, así como los mercados financieros, ya eran débiles antes de la aparición de la pandemia. Por ejemplo, la media de crecimiento mundial fue de $2.8 \%$ en el periodo 2011-2019, la cual es muy baja respecto al $3.4 \%$ que se registró en el periodo 1997-2006. Además, la confianza en el modelo de globalización y multilateralismo actual para el desarrollo ha disminuido, desde la crisis financiera mundial del 2008, o eventos naturales como el tsunami del océano Índico de 2004. A partir de esta referencia, debido a la COVID-19 y sus estrategias de contención, se estimó una reducción de la tasa de crecimiento de la economía mundial de hasta el $1 \%$ y una contracción del 38 \% durante abril a junio. Sin embargo, a finales de marzo del 2020, las caídas de Producto Interno Bruto (PIB) llegaron al 3.8 \% en EE. UU., 9 \% en Europa y 2.1 \% en Japón, que presentan así los albores de un inevitable escenario de recesión mundial (Goldman Sachs, 2020; CEPAL, 2020).

Según la Comisión Económica para América Latina y el Caribe (CEPAL), en la región se pronostican caídas de por lo menos $1.8 \%$ en el PIB y las exportaciones caerían en al menos 10.7 $\%$ en el 2020. Además, prevé que los países sudamericanos serán los más afectados porque sus exportaciones se especializan en productos primarios (hierro, cobre, zinc, aluminio, soja, aceite de soja, entre otros, desde Argentina, Brasil, Chile y Perú) y petróleo, que son más vulnerables a la disminución de precios. Martins (2020) sostiene que las repercusiones económicas son asimétricas porque generan efectos de transmisión en espacio y tiempo, de manera distinta según las características macroeconómicas de cada país $y$ 
microeconómicas de las cadenas globales de producción y consumo. Sin embargo, es posible que las repercusiones iniciales, en los sectores más pobres, sucedan posteriormente en las altas esferas, por la desviación de recursos para hacer frente a la pandemia (Arias et al., 2020).

En el corto plazo, la liquidez de los negocios se vio afectada por los choques de oferta (reducción de las actividades productivas y alteración de las cadenas de suministros por la contención, que podrían estimular la inflación por el incremento de costos) y choques de demanda (reducción de consumo de bienes y servicios por la reacción de los consumidores al aislamiento y distanciamiento social). A mismo tiempo, esto intensificó la volatilidad de los mercados financieros que se suman a la incertidumbre por la intensidad y duración de la pandemia. Las empresas de servicio son las más afectadas (comercio, transporte, servicios empresariales y sociales) y concentran el $64 \%$ de empleados formales. Por otro lado, el 53\% corresponde al empleo informal, que enfrentan una disminución importante de sus ingresos y la pérdida de puestos de trabajo (CEPAL, 2020). Según la Organización Internacional del Trabajo [OIT] (2020), la subsistencia y mantenimiento de la operación de la micro, pequeña y mediana empresa (MIPYME) será difícil.

En América Latina y el Caribe, hasta el momento, los impactos negativos en la economía se resumen en: (1) Reducción de las exportaciones, de las que depende mucho la región y, probablemente, se verá más afectada por la disminución de las actividades económicas de sus principales socios comerciales (China y EE. UU.), debido a la previsión de recesión mundial. (2) Caída de precios de los productos primarios, incluido el mercado petrolero, por una contracción de la demanda mundial, que impacta en los ingresos de las economías regionales dependientes de sus exportaciones. (3) Interrupción de las cadenas globales de valor, principalmente en México y Brasil, donde existen los mayores sectores manufactureros de la región y requieren productos intermedios. (4) Menor demanda de servicios de turismo y comercio, que repercuten en micro y pequeñas empresas (hoteles y restaurantes) y en una cascada de eventos en los ingresos familiares. Estos impactos podrían prolongarse incluso luego de la pandemia (CEPAL, 2020).

En el mediano y largo plazo, respecto al posible escenario de recesión económica mundial, se prevén efectos en la oferta y demanda, quiebre de empresas, reducción de inversión privada, menor crecimiento económico, menor integración en cadenas de valor, así como deterioro de las capacidades productivas y capital humano. El capital de las empresas será afectado porque, generalmente, financian sus inversiones con las ganancias percibidas. Además, la aversión al riesgo y empeoramiento de las condiciones mundiales podrían conllevar a una mayor demanda de activos seguros, menor en activos financieros y depreciación de la moneda de los países. Así, con la disminución de salarios o consiguiente incremento del desempleo, se disminuyen la demanda de bienes y servicios y se acentúan los niveles de pobreza (CEPAL, 2020).

En ese contexto, CEPAL (2020) afirma que los mecanismos de mercados tradicionales podrían no ser suficientes para hacer frente a los efectos posteriores en la economía global. Se vaticina un deterioro en la confianza a proveedores mundiales, que da lugar a lotes de producción más pequeños y pérdidas de economía de 
escala (mayor cantidad de unidades producidas a menor coste). Tal es el caso de China, que ha evidenciado la inestabilidad de un sistema de producción rápido, barato y eficiente cuando el suministro de bienes y servicios se interrumpe, la caída de la oferta de suministros y desplome de las grandes bolsas de valores (Valle et al., 2020). Por ello, Martins (2020) sugiere plantear iniciativas de cooperación multilateral científica y tecnológica; así como políticas económicas nacionales de liquidez o flexibilidad fiscal. Por su parte, CEPAL (2020) propone asumir actividades de planificación con recursos públicos para impulsar y sostener la oferta y la demanda y preservar las capacidades ya instaladas en cada país.

En contraste a los devastadores pronósticos, muchos portales web sostienen que los efectos de la pandemia, ocasionada por el nuevo coronavirus, deben verse como la necesidad de construir una economía global más sostenible. Los enfoques de desarrollo sostenible promueven la adopción de un sistema económico que funcione, tanto para las personas como para el planeta, mediante el aprovechamiento racional y conservación de sus recursos. Inger Anderson, Directora del Programa de las Naciones Unidas para el Medio Ambiente, menciona que la actual pandemia debe verse como una oportunidad para construir una economía diferente, paralelamente con los cambios en nuestros hábitos de producción y consumo, hacia modelos más limpios y sostenibles (Pundir, 2020).

\section{IMPACTOS AMBIENTALES}

El comportamiento y la naturaleza de nuestra sociedad siempre están ligados a aspectos ambientales, mediante interacciones entre las actividades antrópicas y su entorno, que generan modificaciones que pueden acarrear consecuencias positivas y negativas. En la situación actual que el planeta vive, debido al aislamiento social para evitar el contagio de la COVID-19, se han difundido reportes de impactos en algunos aspectos ambientales pocas veces vistos, beneficiosos al medio ambiente y a la biodiversidad.

En el corto plazo, uno de los impactos ambientales positivos, e indirectos de la pandemia, es la reducción de las concentraciones de contaminantes atmosféricos, que se traduce en un aire cada vez más limpio. Todo esto a consecuencia de las medidas de confinamiento de la población, cierre temporal de las diferentes industrias, además de la reducción significativa del transporte público, para reducir el contagio en la población. Es lógico pensar que si no hay actividad industrial, el transporte público $\mathrm{y}$ privado se reduce o restringe, entonces se deja de emitir gases contaminantes a la atmósfera. Este efecto se ha evidenciado en muchos países y muchos medios de comunicación lo han reportado, con imágenes satelitales que demuestran la mejora significativa de la calidad del aire. Por ejemplo, en China se informó sobre la reducción de emisiones durante la cuarentena por la COVID-19, principalmente, por actividades como el transporte y la industria en Beijing, Shanghai, Guangzhou y Wuhan (Wang et al., 2020). Además, según datos de la NASA (Administración Nacional de Aeronáutica y del Espacio) y la ESA (Agencia Especial Europea), durante el bloqueo por la enfermedad, la calidad del ambiente mejoró y las emisiones de dióxido de nitrógeno se redujeron hasta un $30 \%$ en ciudades como 
Wuhan, y países como Italia, España y EE. UU. (Muhammad et al., 2020).

El aislamiento social obligatorio ha traído efectos colaterales beneficiosos para el desplazamiento de las especies de fauna silvestre, debido a la ausencia de la población en áreas protegidas y parques nacionales cerrados por las restricciones impuestas. La reducción de actividades humanas, en espacios naturales, reduce la contaminación atmosférica y acústica, que podría ampliar el uso del hábitat por ciertas especies y reducción de estrés a especies sensibles. En los medios de comunicación se ha informado sobre la presencia de muchas especies en áreas urbanas y rurales, donde hace muchos años no se veían (Corlett et al., 2020). En Perú, también se ha evidenciado la recuperación de las playas del litoral por miles de aves, debido a la ausencia de la población local y turistas, desde que se decretó el aislamiento social.

Durante el transcurso de la pandemia, y probablemente después, muchos aspectos de la forma de vida de la población deberán modificarse. Por ejemplo, para el transporte se reconsideran algunas alternativas con el objetivo de evitar los contagios, como la bicicleta. El uso de la bicicleta se presenta como una alternativa para cumplir con el distanciamiento social y evitar el uso de medios de transporte masivo, que pueden representar focos de infección de la COVID-19. En muchos países, se está impulsando el uso de este medio de transporte, que no solo favorece el distanciamiento social, sino también que genera beneficios físicos que repercuten directamente en la buena salud. En países como Alemania, Bélgica, Países Bajos, Reino Unido, China, Colombia, entre otros, se viene incentivado el uso de este vehículo como una alternativa para frenar el contagio de la COVID-19 (Hermosilla, 2020). Esta alternativa, sin duda, es capaz de conducir a la reducción de la contaminación ambiental, que cada persona genera al movilizarse en cualquier tipo de transporte motorizado. Sin embargo, se debe tener en cuenta que para ello se debe contar con ciclovías habilitadas, que es aún deficiente en países como Perú.

Por otro lado, un problema ambiental, que puede generar la pandemia indirectamente, es la gestión de los residuos sólidos peligrosos generados en hospitales y centros de atención para la COVID-19, como mascarillas, guantes, trajes desechables, entre otros. El uso de mascarillas de forma generalizada por la población ha sido considerado obligatorio en muchos países como una medida de prevención del contagio. Está claro que cada país posee diferentes sistemas de gestión de estos residuos, de acuerdo a su nivel tecnológico; sin embargo, en países de desarrollo intermedio ya se tenían problemas para gestión de residuos de establecimientos de salud, incluso antes de la pandemia. En el escenario de crisis sanitaria, la generación de estos residuos es mayor, intensificando su acumulación. Además, surge la disyuntiva de la clasificación de estos residuos, que generalmente terminaran siendo parte de los residuos sólidos urbanos, y podría suponer una amenaza para los trabajadores de limpieza pública y recojo de residuos. En casos particulares estos tipos de residuos son llevados a combustión incontrolada, lo que podría acrecentar el problema de contaminación atmosférica. Por ello, es necesario hablar de estas prioridades por el gobierno y las entidades de salud, ya que la gestión de residuos debe considerarse esencial para superar la crisis sanitaria (PNUMA, 2020). En 
ese sentido, se deberían generar dispositivos legales sectoriales, dotar de medios económicos para asegurar la prestación de servicios de limpieza, capacitación permanente a los trabajadores de limpieza pública, brindar información a la población permanentemente sobre el manejo de estos residuos, así como supervisión para la disposición final y su confinamiento (Huiman, 2020).

Por último, probablemente, uno de los mayores problemas que la conservación de la biodiversidad y el sector ambiental afronte, en un futuro próximo, sea la reducción de la inversión en proyectos ambientales por parte de los gobiernos. Los proyectos ambientales perderán prioridad porque las inversiones estarán destinadas principalmente a mantener el empleo y los ingresos de la población, mediante salarios, pensiones $y$ beneficios (Bobylev, 2020). Esto es algo que sin duda podría convertirse en una realidad para los países que económicamente se vean disminuidos, luego de superar esta pandemia.

\section{IMPACTOS SOCIALES}

Los impactos sociales ocasionados por las medidas de contención del avance de la COVID-19 están estrechamente ligados a sus impactos económicos. Por ello, antes del estallido de la pandemia, ya se evidenciaba el incremento de los índices de pobreza y extrema pobreza en América Latina y el Caribe, que proyectan repercusiones negativas en la salud, educación y empleo (CEPAL, 2020). Sin embargo, al constituirse como un problema sanitario, se agudizan las consecuencias en los sistemas de salud pública, desaceleración económica que intensifica la brecha entre ricos y pobres, e incluso en la salud mental de la población. Según Pundir (2020), el miedo a la escasez de alimentos está cobrando fuerza en todo el mundo y se evidencia en los reportes de acaparamiento y pánico en la compra de suministros, a pesar de que actualmente aún no hay una preocupación real por la seguridad alimentaria.

Las medidas de aislamiento social están generando efectos en la salud mental de la población. La pandemia no solo trae consigo el riesgo de muerte por la infección de la COVID19, sino también una insufrible presión psicológica (Cao et al., 2020). En lo referente a salud mental, ningún estrato social queda fuera de los efectos psicológicos que podrían originarse como consecuencia de las diferentes situaciones de esta pandemia. Tener familiares, o personas allegadas, infectadas por la enfermedad puede ser un factor de riesgo que incremente los niveles de ansiedad en estudiantes universitarios (Cao et al., 2020) y se podría extrapolar a otros grupos etarios de la población. Durante el aislamiento social podrían presentarse problemas emocionales y hacerse más críticos en la población más vulnerable, como los adultos mayores, que además son más propensos al contagio. La desconexión social y la percepción de aislamiento en adultos mayores, contribuyen a la severidad de los estados de ansiedad y depresión (Santini et al., 2020), que pueden acrecentar el número de víctimas por estos trastornos. Además, un efecto inesperado ocasionado por los medios de comunicación es que podría influir en la salud mental de la población, al enfocarse generalmente en la tragedia que se vive por el COVID-19. Las redes sociales también tienen gran responsabilidad en este fenómeno, porque muchas veces se masifican noticias falsas que contribuyen a 
alarmar a la población y afectar su salud mental. Ante estos problemas de salud mental, se hace considerablemente necesaria la implementación de políticas públicas de respuesta ante futuras emergencias similares (antes, durante y después de los acontecimientos). Por ello, los profesionales de la salud mental son llamados a figurar en primera línea, para brindar soporte a la población necesitada y gestionar estas situaciones extremas (Ornell et al., 2020).

Existen informes sobre el incremento de tasas de violencia doméstica y abuso infantil, por el aislamiento en los hogares. Si bien la incidencia de actos delictivos, en general, se redujeron drásticamente; es necesario prestar atención a las incidencias de actos y denuncias por violencia doméstica y abuso infantil. Se ha demostrado que la violencia familiar y la violencia sexual pueden incrementarse en una crisis de gran magnitud (NZFVC, 2020). Para la población más vulnerable como mujeres, niñas y niños, la medida de aislamiento social y familias encerradas podría significar tener al enemigo en casa (Alon et al., 2020; Plan International, 2020)). En ese sentido, los gobiernos deben adoptar medidas de protección y apoyo terapéutico para que esta población vulnerable no pague el costo más caro durante esta crisis sanitaria (BradburyJones \& Isham, 2020).

La gran expansión de enfermedades emergentes demuestra que ningún país se encuentra a salvo de nuevos agentes infecciosos. Por ello, es importante entender que cada crisis puede brindarnos una valiosa lección. En primera instancia, se debe entender la obligación global de sensibilidad y solidaridad entre países para la cooperación, con la finalidad de evitar una mayor crisis económica y de vidas humanas (Arias et al., 2020). Para Valle et al. (2020), la crisis ocasionada por la COVID-19 representa un elemento disruptivo del proceso de globalización, ya que el modo de tratarla requiere profundos cambios en la estructura social de todo el mundo. Por ejemplo, se destaca la necesidad de cambios en el actual modelo de desarrollo económico neoliberal, por uno más integrador con el medio ambiente y todos los miembros de la sociedad global (acorde a los objetivos de Desarrollo Sostenible). En este contexto, según Valle et al. (2020) y BBC News Mundo (2020), queda demostrada la responsabilidad del ser humano en la deforestación progresiva que aísla a la fauna silvestre, el consumo de animales salvajes, incluso la producción intensiva de alimentos, así como el alto nivel demográfico, que favorecieron la circulación del virus. Además, se propone como punto de partida la prohibición de consumir la fauna silvestre.

En el aspecto sanitario, existen reportes de crisis en los sistemas de salud, negligencias médicas y hasta casos de xenofobia en la atención de casos severos. Sin embargo, es necesario resaltar la importancia del personal del sector salud y de otras personas que realizan servicios clave (BBC News Mundo, 2020). Por otro lado, el portal Infosalus (10/04/2020) da a conocer que la Sociedad Española de Médicos y Atención Primaria (Semergen) considera que esta es una oportunidad para mejorar el uso de la telemedicina, por ejemplo, en beneficio de pacientes con enfermedades cardiovasculares o renales, que permitiría transformar la relación médico-paciente y potenciar la posibilidad de llegar a más pacientes. Además, se percibe el refuerzo del empoderamiento y autocuidado de los pacientes, como el realizar actividad física o 
consumir menos sal, impulsado, tal vez, por el miedo a experimentar una complicación de su enfermedad de base y verse obligados a acudir a los servicios de emergencia de los hospitales.

En la población general de todo el mundo, se ha promovido un sentimiento de unidad. Adicionalmente, el distanciamiento social físico ha sido motivo para reconectar y fortalecer las relaciones con el círculo familiar y amical, mediante llamadas telefónicas o videollamadas. De igual manera, este sentimiento ha promovido actos de bondad en todo el mundo, como entrega de alimentos y medicamentos a poblaciones vulnerables, donaciones de dinero, eventos virtuales con mensaje esperanzador, y hasta solidaridad de pasatiempos y compañía a través de redes sociales, para combatir los efectos del autoaislamiento en la salud mental de las personas. Muchas personas ocupan su tiempo en capacitarse o capacitar al público, hacen recorridos virtuales por museos o solo dan rienda suelta a su creatividad y aventurarse en nuevos pasatiempos (como leer, cocinar, pintar, ejercitarse, etc.), para combatir el aburrimiento. Curiosamente, también existen reportes de mayor participación de hombres en las cocinas, que promueven, sutilmente, la equidad de género durante el periodo de aislamiento obligatorio en los hogares (BBC News Mundo, 2020).

El aislamiento y distanciamiento social obligatorio han permitido el tránsito del trabajo y la educación al ámbito digital, que constituye un aliciente para el mercado de tecnologías. Además, esto ha hecho posible continuar las relaciones económicas y sociales, como un soporte de la comunicación interpersonal y actividades de entretenimiento. Incluso se prevé que el teletrabajo prevalecerá en más industrias y regiones después de la pandemia, porque generan ventajas para las empresas más avanzadas tecnológicamente respecto a las que no lo están. Sin embargo, es necesario considerar que el incremento de tecnologías digitales podría exacerbar las desigualdades sociales debido al acceso y la conectividad a estas (CEPAL, 2020).

Muchos portales web se enfocan en tratar de visibilizar las oportunidades para la agricultura, que puede acarrear la coyuntura actual. Por ejemplo, Andina (2020) publicó una entrevista a Gabriel Amaro, Director Ejecutivo de la Asociación de Gremios Productores Agrarios (AGAP) de Perú, quien menciona que los países fortalecieron sus cadenas de producción agrícola, industrialización $\mathrm{y}$ comercialización de productos alimenticios. Una mayor demanda de volúmenes de frutas (cítricos, arándanos y similares) y hortalizas puede ser explicada por la creciente tendencia del deseo de una mejor alimentación de la gente, que optan por productos frescos que ayuden a mejorar sus defensas. Por su parte, Pundir (2020) sostiene que, probablemente, la pandemia podría desencadenar algunas tendencias, como la búsqueda de autosuficiencia alimentaria o un sistema alimentario hiperlocal, quizá como respuesta a la ansiedad sobre el futuro incierto. Sin embargo, tratar de preservar el alto nivel de dependencia de la población, a necesidades básicas como la alimentación, podría ser contraria a los objetivos de desarrollo sostenible. Por ello, es destacable que, por ejemplo en EE. UU., las búsquedas online sobre "Agricultura doméstica” o “Cómo criar pollos?" hayan aumentado en $50 \%$ y $75 \%$ respecto al mes anterior, que evidencia una preocupación y la magnitud de los cambios que se deberían promover. Incluso, se afirma que las 
consecuencias de la pandemia podrían no ser tan malas para los pequeños agricultores porque podría ocurrir un cambio del consumo hacia alimentos locales bajo el slogan "de la chacra a la mesa", la que también puede construir mayor resiliencia en las sociedades y economías a las vicisitudes de la globalización.

El escenario actual y el pronóstico de escasez alimentaria están concientizando a muchas personas sobre la adopción de estrategias más sostenibles para su subsistencia, como la autosuficiencia para cultivar sus propios alimentos mediante agricultura urbana. En esta coyuntura, este concepto está ganando fuerza y no necesita gran extensión de terreno (espacios urbanos e incluso en apartamentos pequeños son adecuados), pero con la técnica correcta. Este tipo de agricultura tiene la capacidad de mejorar la seguridad alimentaria y la nutrición de la población, permitir la elección de alimentos libres de contaminantes, reducir los impactos del cambio climático e incluso disminuir el estrés. Sin embargo, el interés real de cambio de las políticas y la población solo se verán cuando concluya el bloqueo, pero es bueno difundir las iniciativas (Pundir, 2020).

\section{CONCLUSIONES}

Algunas medidas económicas paliativas para la pandemia se resumen en cooperación internacional y multilateral para diseñar instrumentos técnicos y financieros (préstamos con bajo interés y aplazamiento de deudas) para apoyar a los países más afectados. La CEPAL recomienda un estímulo de gasto fiscal suficiente para fortalecer los servicios de salud, así como para proteger los ingresos y los empleos. Los bancos centrales deben asegurar la liquidez de las empresas para garantizar el funcionamiento $\mathrm{y}$ estabilidad del sistema financiero, para evitar la interrupción de las cadenas de pago. En esa línea, en el aspecto social se propone reforzar los sistemas de protección social para apoyar a las poblaciones vulnerables, mediante transferencias efectivas y créditos sin interés a empresas para el pago de salarios, aplazamientos de pago de deudas, alquileres y servicios básicos a personas de bajos ingresos, durante la pandemia. Además, se debe garantizar la provisión de suministros esencialmente alimenticios y de salud. Sin duda, los impactos ambientales positivos predominan sobre los negativos. Se resalta la disminución de las emisiones propias del transporte y las industrias que mejoran la calidad del aire; además de los beneficios para el desplazamiento de la fauna silvestre, debido a la restricción de tránsito de la población.

Desde una perspectiva objetiva, desde hace varios años ya se percibían atisbos de la insostenibilidad del actual sistema económico global en el largo plazo. Ante esto, la crisis sanitaria por la COVID-19 ha evidenciado la fragilidad de este sistema y del actual modelo de desarrollo. Por ello, constituye una lección y oportunidad para la creación de una sociedad más justa y solidaria en todos sus ámbitos. Para lo cual, la comunidad mundial debe considerar reformular profundamente el sistema de globalización, hacia un modelo de desarrollo sostenible que incluya el abordaje de las desigualdades entre países y grupos sociales, así como el deterioro medioambiental. La explosión del nuevo coronavirus enseña que los flujos biológicos deben ser tomados en cuenta, dentro de un nuevo paradigma de globalización porque la especie humana, como parte del sistema ecológico, se ha vuelto muy vulnerable y dependiente, a diferencia de las 
otras especies que viven en perfecto equilibrio con la naturaleza.

\section{Agradecimientos}

Los autores agradecen al programa de doctorado en Ciencias para el Desarrollo Sustentable de la Universidad Nacional Toribio Rodríguez de Mendoza de Amazonas, financiado en el marco del proyecto FondecytBanco Mundial “Doctorados en Áreas Estratégicas y Generales" con contrato $N^{\circ} 003-$ 2018-FONDECYT/BM-PROGRAMAS DE DOCTORADOS EN ÁREAS ESTRATÉTICAS Y GENERALES.

\section{Referencias bibliográficas}

Andina (2020, 08 de mayo). Perú se consolida como uno de los principales abastecedores de alimentos del mundo. Recuperado el 10 de mayo del 2020, de https://andina.pe/agencia/noticia-peru-se-consolidacomo-uno-de-principales-abastecedores-alimentosdel-mundo-795686.aspx

Alon, T. M., Doepke, M., Olmstead-Rumsey, J., \& Tertilt, M. (2020). The impact of COVID-19 on gender equality. National Bureau of Economic Research. https://doi.org/10.3386/w26947

Arias, Y. E., Pinzón, M. V., Zúñiga, L. F., Zambrano, E. D., Saavedra-Torres, J. S. (2020). Estragos socioeconómicos de un pensamiento filosófico de salud pública por el virus COVID-19. Virus COVID19 y Economía, p. 2.

BBC News Mundo (2020, 23 de marzo). Coronavirus: 5 cosas positivas que han surgido de la crisis por la pandemia. Recuperado el 10 de mayo del 2020, de https://www.bbc.com/mundo/noticias-51986136

Bobylev, S. N. (2020). Environmental consequences of COVID-19 on the global and Russian economics. Population and Economics, 4(2): 43-48. https://doi.org/10.3897/popecon.4.e53279

Cao, W., Fang, Z., Hou, G., Han, M., Xu, X., Dong, J., \& Zheng, J. (2020). The psychological impact of the COVID-19 epidemic on college students in China. Psychiatry Research, 112934. https://doi.org/10.1016/j.psychres.2020.112934
CEPAL (Comisión Económica para América Latina y el Caribe) (2020, 03 de abril). América Latina y el Caribe ante la pandemia del COVID-19. Efectos económicos y sociales. Informe Especial COVID-19 $N^{\circ} 1$. Recuperado el 08 de mayo del 2020, de https://repositorio.cepal.org/bitstream/handle/11362 /45337/4/S2000264_es.pdf

Corlett, R. T., Primack, R. B., Devictor, V., Maas, B., Goswami, V. R., Bates, A. E., Koh, L. P., Regan, T. J., Loyola, R., \& Pakeman, R. J. (2020). Impacts of the coronavirus pandemic on biodiversity conservation. Biological Conservation, 246:108571. https://doi.org/10.1016/j.biocon.2020.108571

Goldman Sachs. (2020, 24 de marzo). Roaring into recession. Top of Mind. $N^{\circ} 87$. Recuperado el 08 de mayo del 2020, de https://www.goldmansachs.com/insights/pages/roar ing-into-recession-f/report.pdf

Hermosilla, D. (2020, 14 de abril). ¿Distanciamiento social en el transporte público? Bicicleta vuelve a aparecer como alternativa de movilización. La Tercera. Recuperado el 10 de mayo del 2020, de https://www.latercera.com/quepasa/noticia/distanciamiento-social-en-eltransporte-publico-bicicleta-vuelve-a-aparecercomo-alternativa-demovilizacion/5S2PW7NXOZEGVBW3IP7ESE7H74/

Huiman. A. (2020, 02 de mayo). Gestión de residuos sólidos en tiempos de COVID-19. Recuperado el 08 de mayo del 2020, de https://www.pucp.edu.pe/climadecambios/entrevist as/gestion-de-residuos-solidos-en-tiempos-decovid-19/

Infosalus. (2020, 04 de abril). Los efectos "positivos" para la salud de la pandemia por COVID-19. Recuperado 15 de mayo del 2020, de https://www.infosalus.com/ asistencia/noticia-efectos-positivos-salud-pandemiacovid-19-20200410103844.html

Martins, E. (2020). Novo coronavírus e seus impactos económicos no mundo. Boletim de Conjuntura, 1(2): 39-42. http://doi.org/10.5281/zenodo.3761708

Muhammad, S., Long, X., and Salman, M. (2020). COVID19 pandemic and environmental pollution: A blessing in disguise? Science of The Total Environment, 728: 138820. https://doi.org/10.1016/j.scitotenv.2020.138820

New Zealand Family Violence Clearinghouse (NZFVC). (2020). Preventing and responding to family, whānau and sexual violence during COVID-19. Recuperado el 08 de mayo del 2020, de https://nzfvc.org.nz/COVID19/preventing-responding-violence-COVID-19 
OMS. (2020). Nuevo coronavirus 2019. Recuperado el 08 de mayo del 2020, de https://www.who.int/es/ emergencies/diseases/novel-coronavirus-2019

OIT (Organización Internacional del Trabajo). (2020, 18 de marzo). El COVID-19 y el mundo del trabajo: Repercusiones y respuestas. Recuperado el 10 de mayo del 2020, de https://www.ilo.org/wcmsp5/ groups/public/---dgreports/---

dcomm/documents/briefingnote/wcms_739158.pdf

Ornell, F., Schuch, J. B., Sordi, A. O., \& Kessler, F. H. P. (2020). "Pandemic fear" and COVID-19: Mental health burden and strategies. Brazilian Journal of Psychiatry, 42(3): 232-235. https://doi.org/10.1590/1516-4446-2020-0008

Plan International. (2020, 15 de abril). En cuarentena con el enemigo. Recuperado 12 de mayo del 2020, de https://www.planinternational.org.pe/blog/encuarentena-con-el-enemigo

PNUMA. (2020, 24 de marzo). La gestión de residuos es un servicio público esencial para superar la emergencia de COVID-19. UNEP - UN Environment Programme. Recuperado el 12 de mayo del 2020, el http://www.unenvironment.org/es/noticias-y- reportajes/comunicado-de-prensa/la-gestion-deresiduos-es-un-servicio-publico-esencial

Pundir, P. (2020). Ecoportal: La mayor enseñanza de la pandemia: cultiva tu propia comida. Recuperado de https://www.ecoportal.net/econciencia/autoconsum o/cultuva-tu-comida/?cn-reloaded $=1$

Sánchez, N. A. (2010). Pobreza, Medio Ambiente y Desarrollo Sostenible. Nómadas. Critical Journal of Social and Juridical Sciences, 26(2). http://www.redalyc.org/articulo.oa?id=18118916003

Santini, Z. I., Jose, P. E., York Cornwell, E., Koyanagi, A., Nielsen, L., Hinrichsen, C., Meilstrup, C., Madsen, K. R., \& Koushede, V. (2020). Social disconnectedness, perceived isolation, and symptoms of depression and anxiety among older Americans (NSHAP): A longitudinal mediation analysis. The Lancet Public Health, 5(1): e62-e70. https://doi.org/10.1016/S24682667(19)30230-0

Wang, P., Chen, K., Zhu, S., Wang, P., \& Zhang, H. (2020). Severe air pollution events not avoided by reduced anthropogenic activities during COVID-19 outbreak. Resources, Conservation and Recycling, 158: 104814. https://doi.org/10.1016/j.resconrec.2020.104814 